\title{
1070. Effects of two different mechanical ventilation strategies on lung conditions after experimental ARDS following blunt chest trauma and pulmonary contusion in pigs
}

\author{
S Hammermüller ${ }^{1 *}$, N Carvalho², S Huckauf ${ }^{1}$, S Kobelt ${ }^{1}$, M Baunack ${ }^{1}$, K Noreikat $^{1}$, A Beda ${ }^{2}$, A Reske $^{3}$, H Wrigge $^{1}$, \\ AW Reske ${ }^{1}$
}

From ESICM LIVES 2014

Barcelona, Spain. 27 September - 1 October 2014

\section{Introduction}

Pulmonary contusion (PC) is common after blunt chest trauma, leads to inhomogeneous lung injury and can result in acute respiratory distress syndrome (ARDS) [1]. Strategies for mechanical ventilation (MV) with different physiological rationales and approaches to positive endexpiratory pressure (PEEP) and tidal volume (TV) adjustment are proposed [1-3].

\section{Objective}

To study the effects of the ARDSnetwork lower PEEP (ARDSnet) [1] and the Open Lung Concept (OLC) [2] strategies for MV on lung ventilation and function over 24 hours in pigs after experimental PC.

\section{Methods}

Pigs $(n=16)$ were anesthetized, tracheotomized and received MV. Catheters were placed aseptically. Cefuroxime $750 \mathrm{mg}$ was given IV q6h. Unilateral PC was induced by a $10 \mathrm{~kg}$ weight dropped from $1.85 \mathrm{~m}$ height on a predefined location of the right chest. Chest tubes were inserted on both sides. Conditions comparable to an ICU were established. At 90 min after PC (post-PC) pigs were randomized to 24 hours of MV using ARDSnet $(n=8)$ or OLC $(n=8)$. Pressure controlled MV in the OLC group involved: an initial recruitment maneuver (50 $\mathrm{cmH}_{2} \mathrm{O}, 10$ breaths), respiratory rate $80 / \mathrm{min}, \mathrm{I} / \mathrm{E}$ $2: 1, \mathrm{TV}<6 \mathrm{ml} / \mathrm{kgBW}$, positive inspiratory pressure
(PIP) $\leq 30 \mathrm{cmH}_{2} \mathrm{O}$. Total PEEP of approx. $19 \mathrm{cmH}_{2} \mathrm{O}$ resulted from development of intrinsic PEEP on top of external PEEP of $10 \mathrm{cmH}_{2} \mathrm{O}$. Cardiorespiratory, gas exchange and extra-vascular lung water (EVLW, singleindicator transpulmonary thermodilution) parameters were measured. Electrical impedance tomography was used to assess changes in lung ventilation (Vent). Vent was calculated as the number of pixels showing an impedance change of $>15 \%$ of the global impedance change and expressed as \% of baseline. Data are given as median and interquartile $\left(25^{\text {th }}-75^{\text {th }}\right)$ range. Mann-Whitney-tests and General Linear Model statistics were used.

\section{Results}

Cardiorespiratory conditions were stable without significant between-group differences at pre-PC and post-PC. At 24 hours after randomization PEEP was significantly lower in ARDSnet $\left(8(5-10) \mathrm{cmH}_{2} \mathrm{O}\right)$ vs. OLC 19 (17-21) $\left.\mathrm{cmH}_{2} \mathrm{O}\right) . \mathrm{PaO}_{2} / \mathrm{FIO}_{2}$ (477 (296-514) vs. 87 (72-118) $\mathrm{mmHg}$ ) and static compliance were significantly higher in OLC (Tab.1). Intrapulmonary shunt (28 (27-36) vs. 11 (8-18) \%), $\mathrm{PaCO}_{2}$ (46 (43-63) vs. 43 (33-45) $\mathrm{mmHg}$ ), TV (7 (7-7) vs. 5 (5-6) $\mathrm{ml} / \mathrm{kg} \mathrm{BW),} \mathrm{driving} \mathrm{pressure} \mathrm{(deltaP)}$ and EVLW were all significantly higher in ARDSnet after 24 hours, whereas Vent was significantly lower (Table 1). The difference in PIP (OLC 29 (27-30) $\mathrm{cmH}_{2} \mathrm{O}$ vs. ARDSnet $\left.34(29-38) \mathrm{cmH}_{2} \mathrm{O}\right)$ was not statistically significant. 
Table 1

\begin{tabular}{llllllllll}
\hline & Group & Pre-PC & Post-PC & 4 hrs. & 8 hrs. & 12 hrs. & 16 hrs. & 20 hrs. & 24 hrs. \\
\hline $\begin{array}{l}\text { Compliance } \\
\text { (ml/cmH2O) }\end{array}$ & ARDSnet & $25(23-30)$ & $18(17-20)$ & $18(16-19)$ & $17(16-19)$ & $17(16-19)$ & $16(13-17)$ & $15(14-16)$ & $13(11-17)$ \\
\hline & OLC & $25(21-34)$ & $17(15-18)$ & $15(16-19)$ & $19(14-23)$ & $18(17-23)$ & $21(17-26)$ & $23(17-26)$ & $21(17-24)$ \\
\hline EVLW (ml/kg) & ARDSnet & $333(299-377)$ & $344(285-379)$ & $280(254-347)$ & $338(368-372)$ & $368(283-411)$ & $361(334-398)$ & $346(339-365)$ & $375(350-410)$ \\
\hline & OLC & $333(283-345)$ & $345(284-411)$ & $320(275-378)$ & $327(268-365)$ & $318(276-326)$ & $327(273-372)$ & $311(269-359)$ & $321(290-397)$ \\
\hline $\begin{array}{l}\text { Ventilated } \\
\text { area (Vent\%) }\end{array}$ & ARDSnet & 100 & $90(72-94)$ & $81(77-90)$ & $77(73-83)$ & $80(76-92)$ & $79(76-92)$ & $76(71-87)$ & $77(72-90)$ \\
\hline & OLC & 100 & $90(86-98)$ & $93(70-103)$ & $94(85-100)$ & $98(88-110)$ & $99(93-116)$ & $99(93-116)$ & $104(94-167)$ \\
\hline $\begin{array}{l}\text { deltaP } \\
(\mathrm{cmH2O})\end{array}$ & ARDSnet & $16(13-22)$ & $21(19-23)$ & $20(19-23)$ & $21(19-25)$ & $22(19-25)$ & $22(19-26)$ & $22(17-28)$ & $24(19-28)$ \\
\hline & OLC & $21(17-24)$ & $26(21-27)$ & $9(8-11)$ & $10(8-11)$ & $10(8-11)$ & $10(8-11)$ & $10(9-11)$ & $10(9-11)$ \\
\hline
\end{tabular}

\section{Conclusions}

OLC ventilation better fulfilled common criteria for lung protection, because it facilitated MV with lower TV, deltaP, less edema (EVLW) and better lung function. It also prevented progressive derecruitment (decrease in Vent) during lung protective ventilation.

\section{Grant acknowledgement}

CNPq, CAPES, FAPEMIG, B.Braun-Foundation and DFG.

\section{Authors' details}

${ }^{1}$ University Leipzig, Department of Anesthesiology and Intensive Care Medicine, Leipzig, Germany. ${ }^{2}$ University of Minas Gerais, Department of Electronic Engineering, Federal, Belo Horizonte, Brazil. ${ }^{3}$ Fachkrankenhaus Coswig, Coswig, Germany.

Published: 26 September 2014

\section{References}

1. Anonymous: NEJM 2000

2. Schreiter D, Reske A, Stitchert B, Seiwerts M, Bohm SH, Kloeppel R, Josten C: Alveolar recruitment in combination with sufficient positive endexpiratory pressure increases oxygenation and lung aeration in patients with severe chest trauma. Crit Care Med 2004, 32(4):968-975.

3. Andrew PL: J Trauma 2013.

\section{doi:10.1186/2197-425X-2-S1-P86}

Cite this article as: Hammermüller et al:: 1070. Effects of two different mechanical ventilation strategies on lung conditions after experimental ARDS following blunt chest trauma and pulmonary contusion in pigs.

Intensive Care Medicine Experimental 2014 2(Suppl 1):P86.

\section{Submit your manuscript to a SpringerOpen ${ }^{\circ}$ journal and benefit from:}

- Convenient online submission

- Rigorous peer review

- Immediate publication on acceptance

- Open access: articles freely available online

- High visibility within the field

- Retaining the copyright to your article

Submit your next manuscript at $\boldsymbol{s p r i n g e r o p e n . c o m ~}$ 